\title{
Relación entre el nivel de actividad física y sedentarismo, sobrepeso y calidad de vida relacionada con la salud en niños asmáticos en edad escolar: un estudio exploratorioenSevilla
}

\author{
Relationship between the level of physical activity and sedentary, overweight and health-related quality \\ of life in scholar-age asthmatic children: an explanatory study in Seville
}

\author{
Borja del Pozo-Cruz, Jesús del Pozo-Cruz, Francisco Javier González Limones, Rosa María Alfonso Rosa \\ Universidad de Sevilla
}

\begin{abstract}
Resumen: Objetivo. En este estudió se pretendió un objetivo doble; 1) Constatar las diferencias entre niños asmáticos con sobrepeso y normopeso en cuanto a CVRS y cantidad de actividad física y sedentarismo y 2) Determinar la relación existente entre cantidad de actividad física, sedentarismo e IMC y su influencia en la CVRS de esta población en Sevilla. Método. Se usó un diseño de cohorte que incluyó a 69 niños en edad escolar con asma persistente controlado. Las medidas incluidas en el estudio fueron: el cuestionario PAQL(S), para evaluar la CVRS de los niños y el cuestionario IPAQ-A, para evaluar la cantidad de actividad física y sedentarismo de los niños. También se calculó el IMC y se determinó si el participante estaba en normopeso o sobrepeso. Resultados. Los niños con sobrepeso obtuvieron peores valores en CVRS e IPAQ-A que sus pares con normopeso. Además se observaron correlaciones positivas entre el IMC y la cantidad de sedentarismo y negativas entre el IMC y también entre éste y el valor en las escalas de valoración de la CVRS. Conclusión. El IMC afecta negativamente a la CVRS a los niños asmáticos en edad escolar.
\end{abstract}

Palabras clave;niños asmáticos, enfermedad crónica, hábitos de vida, ejercicio físico.

Abstract: Objective. The aim of this study was: 1) To determine the differences between normal-weight asthmatic children and overweight asthmatic children in terms of HRQoL and amount of physical activity and sedentary and 2) To determine the relationship between amount of physical activity, sedentary and BMI and their influence on the HRQoL of this population in Seville. Method. We used a cohort design study that included 69 school-age children with controlled persistent asthma. The measures included in the study were: the questionnaire PAQL (S), to assess the HRQoL of children and the IPAQ-A questionnaire to assess the amount of physical activity and sedentary lifestyle of children. BMI was also calculated and determined if the participant was of normal-weight or overweight. Results. Overweight children scored worse on HRQoL and IPAQ-A values than their peers with normalweight. Positive correlations were also observed between BMI and the amount of sedentary lifestyle. Negative correlations were observed between BMI and the amount of physical activity and also negative correlations were observed between BMI and HRQoL. Conclusion. BMI negatively affect HRQoL in school- age asthmatic children.

Key words: asthmatic children, chronic disease, lifestyles, physical exercise.

\section{Introducción}

Asma y sobrepeso/obesidad han liderado los problemas de salü pública durante las últimas décadas debido a su alta tasa de prevalencia y las consecuencias socio-económicas que presentan (Beuther, Weiss \& Sutherland, 2006). Según el estudio internacional de asma y alergia en niños (ISAAC) realizado en España (Carvajal-Uruena et al., 2005), la prevalencia de síntomas asmáticos en niños de 13-14 años se ha mantenido constante durante los últimos años mientras que en niños de 6-7 años existe un aumento significativo (Carvajal-Ureña, García-Marcos, Busquets-Monge, Morales Sánchez-Varela \& García DeAndoain, 2005). La evidencia científica disponible sugiere que existe una relación entre sobrepeso/obesidad y asma en edad escolar (Ahmad et al., 2009) aunque la dirección de dicha relación no está clara (Gilliland et al., 2003). Es decir, no está claro si el sobrepeso puede ser causante del asma o viceversa. En niños y niñas con sobrepeso u obesidad los síntomas de asfixia pueden ser debidos al incremento del trabajo respiratorio (Schachter, Peat \& Salome, 2003). Por otro lado, los niños y niñas con asma pueden ver disminuida su actividad física $\mathrm{y}$, a su vez, algunos fármacos empleados en su tratamiento pueden inducir una ganancia en el peso corporal (Lobstein, Baur \& Uauy, 2004). En este sentido, la investigación ha examinado una gran variedad de posibles mecanismos subyacentes de la conexión entre obesidad y asma, incluyendo vínculos genéticos (Beuther, et al., 2006), factores ambientales que impiden la realización de actividad física (Brisbon, Plumb, Brawer \& Paxman, 2005) o marcadores biológicos (Story, 2007). A pesar de los esfuerzos realizados por la comunidad científica, las rutas exactas por las que asma y sobrepeso/obesidad se vinculan están aún por determinar. Si bien

Fecha recepción: 03-11-11- Fecha envío revisores: 03-11-11 - Fecha de aceptación: 07-12-11 Correspondencia: Borja del Pozo-Cruz

C/Pirotecnia s/n

41013 Sevilla (Sevilla)

Email: bpozo@us.es parece claro que la pérdida de peso mejora la función respiratoria, reduce los síntomas propios de la enfermedad y la dependencia de medicamentos para el control del asma se minimiza (Ford, 2005), hasta nuestro conocimiento, no está del todo claro la relación existente entre el nivel de actividad física, la obesidad y la calidad de vida relacionada con la salud (CVRS) en pacientes asmáticos en edad escolar. Además se adolecen de datos españoles en este sentido. De hecho, el objetivo este estudio piloto fue doble: 1) Constatar las diferencias entre niños asmáticos con sobrepeso y normopeso en cuanto a CVRS y cantidad de actividad física y sedentarismo determinar y 2) Determinar la relación existente entre cantidad de actividad física, sedentarismo e Índice de Masa Corporal (IMC) y su influencia en la CVRS de pacientes asmáticos en edad escolar en Sevilla.

\section{Método}

\subsection{Diseño y muestra}

Se usó un diseño de cohorte que incluyó a 69 niños en edad escolar con asma persistente de leve a moderado controlados con una edad comprendida entre los 7 y 17 años. Todos los participantes fueron identificados y reclutados en la consulta de Neumología de un Hospital de la ciudad de Sevilla, España durante el periodo de Febrero a Junio de 2010. Se usó un muestreo por conveniencia no probabilístico, dada la especificidad de la muestra. Los criterios de inclusión para este estudio fueron los que siguen: tener entre 7 y 17 años (Bateman et al., 2008); tener asma de leve a moderado persistente controlado de al menos 6 meses según criterios de la Iniciativa Global para el Asma (GINA) (Bateman, et al., 2008) y presentar valores basales superiores al $80 \%$ en los coeficientes FEV1, FVC y FEV1 basal antes del estudio (considerados coeficientes correspondientes a una función de ventilación normal). Los participantes fueron excluidos del estudio si padecían alguna afección que le impidiese realizar las evaluaciones requeridas en la investigación. Cuidadores principales y participantes fueron informados detalla- 
damente de la investigación de forma escrita y oral y firmaron un consentimiento informado aceptando formar parte de la misma. El protocolo de recogida de datos fue archivado, y a cada participante se le asignó una clave de tal modo que no podía relacionarse la muestra e información obtenida con la identidad del sujeto.

\subsection{Medidas}

Las características socio-demográficas (edad y sexo) y antropométricas (talla, peso e IMC) fueron recogidos en una hoja autodiseñada para tal fin. La edad y el sexo fueron preguntados de forma oral mientras que para la determinación del peso se usó una báscula SECA 797 (SECA, Hamburgo, Alemania), con precisión de \pm 100 gr. La forma de realizar la medida está estandarizada, permaneciendo el individuo de pie en el centro de la plataforma, desprovisto de ropa, y con el peso distribuido por igual en ambos pies y sin apoyos. La talla se obtuvo con tallímetro Holtain (Holtain Ltd., Dyfed, Reino Unido), también en la forma estandarizada. El sujeto permaneció de pie, con los talones juntos, brazos a lo largo del cuerpo y las nalgas y la espalda apoyadas sobre la escala y con la cabeza situada en el plano de Frankfort. Para determinar el IMC se utilizó la fórmula: Peso (Kg) / Estatura ${ }^{2}(\mathrm{~m})$, de Quételet (Sucharda, 1989). Los sujetos fueron clasificados en función de si tenían normopeso o sobrepeso. Para definir el grupo de sobrepeso se tomaron los valores de IMC superiores al percentil 85 de la distribución de referencia, en función de la edad y el sexo. Se determinó el nivel de actividad física y sedentarismo (horas sentado) a través del Cuestionario Internacional de Actividad Física (IPAQ) en su versión corta española validada para adolescentes (IPAQ-A) (Hagstromer et al., 2008). El IPAQ mide el nivel de actividad física a través de preguntas en cuatro dominios: ocupacional, doméstico, de transporte y del tiempo libre. El indicador de actividad física se expresa tanto de manera continua, en MET-minutos/semana (este es el indicador usado en el presente manuscrito), como de manera categórica, clasificando el nivel de actividad física en bajo, moderado o alto. Los METs son una forma de calcular los requerimientos energéticos, son múltiplos de la tasa metabólica basal y la unidad utilizada, MET-minuto, se calcula multiplicando el MET correspondiente al tipo de actividad por los minutos de ejecución de la misma en un día o en una semana, es así como en el presente trabajo se expresa en MET-minuto/semana. Para evaluar la CVRS se usó el Cuestionario de Calidad de Vida en niños con asma en su versión española con actividades estandarizadas (PAQLQ(S)) (Tauler, Ferrer, Vall \& Alonso, 2002). El cuestionario PAQLQ(S) presenta 23 ítems repartidos en 3 dimensiones que comprenden: Síntomas (10 ítems), Función emocional (8 ítems) y Limitación de actividades (5 ítems). Este cuestionario arroja un índice global de CVRS que se obtiene de la media de la puntuación en los diferentes ítems que lo componen, los cuales presentan una escala de 1 a 7 ( 1 peor estado posible a 7 mejor estado posible de dicho ítem). El punto de corte en el PAQLQ(S) ha sido determinado en 5,90 puntos (por debajo del cual se considera que los pacientes presentan un nivel de CVRS reducido respecto a sus pares) (CanoGarcinuno et al., 2007).

Tabla 1. Características de los partici pantes en el estu dio (n=69)

\begin{tabular}{ll}
\hline Medidas principales & Media (DE) o Porcentaje (\%) \\
\hline Edad (años) & $14.45(1.63)$ \\
Sexo (\%) & 53.60 (Chi cos); 46.40 (Chi cas) \\
Sobrepeso/Obesidad & $29(\mathrm{~S}) ; 40(\mathrm{~N})$ \\
Talla (cm) & $161.57(9.97)$ \\
Peso (Kg.) & $62.28(9.44)$ \\
IMC (Kg./ $\left.\mathrm{cm}^{2}\right)$ & $23.19(3.34)$ \\
PAQL(S) & $5.24(1.13)$ \\
Tiempo total de actividad (min.) & $116.38 \pm(142.16)$ \\
Tiempo total sentado (min.) & $114.66 \pm(69.61)$ \\
\hline *Valores expresdos como Media \pm (DE) o porcentaje; S: Sí; N: No; IMC: Índice de Masa Corporal; \\
PAQL (S): Cuestionario de calidad de vida relacio nado con la salud en niños asmáticos.
\end{tabular}

PAQL (S): Cuestionario de calidad de vida relacio nado con la salud en niños asmáticos.

\subsection{Procedimiento}

Una vez se obtuvo el consentimiento informado y se verificaron los criterios de inclusión, los sujetos fueron citados en días posteriores durante el periodo arriba indicado para su evaluación en una sola sesión. Fue habilitada una sala del hospital para la investigación. En primer lugar se evaluaron las características socio-demográficas antropométricas básicas que incluían la talla y el peso para posteriormente calcular el IMC mediante fórmula matemática arriba indicada. Finalmente, se les pidió a los sujetos que rellenaran los cuestionarios seleccionados para esta investigación (que incluían el IPAQ versión corta para evaluar el nivel de actividad física y sedentarismo y el PAQLQ para evaluar la CVRS de los pacientes) en presencia de un técnico externo al equipo investigador que resolvía cualquier duda que pudiera surgir respecto a los cuestionarios.

\subsection{Análisis estadístico}

Todos los análisis estadísticos fueron realizados con el paquete estadístico SPSS 18.0. Se estableció un nivel de significación estadística de $p<.05$ en todos los análisis realizados. Se realizó un análisis descriptivo de todas las variables incluidas en el estudio. La estadística descriptiva fue presentada como media y DE. La normalidad de los datos fue testada inicialmente usando el test de Kolgomorov-Smirnov con corrección de Lillifors. Los resultados de dicha prueba sugirieron el uso de un análisis paramétrico. Las diferencias entre grupos fueron analizadas mediante el test $\mathrm{t}$ de Student. Para estandarizar las puntuaciones, la diferencia entre las puntuaciones en bruto de los niños con sobrepeso y la puntuación media del grupo de referencia (niños en normopeso) fue calculada. Estas diferencias fueron entonces divididas por la DE del grupo control. Estas puntuaciones estándares (z-scores) expresan la distancia individual desde el grupo de referencia en términos de distribución (tamaño del efecto). Así, cualquier puntuación igual a la media del grupo de referencia será equivalente a un tamaño del efecto igual a 0 . Los valores positivos o negativos indican un individuo que cae por encima o por debajo de la media respectivamente. Se usó una el coeficiente de correlación de Pearson para determinar el grado de correlación entre las diferentes variables del estudio. Se realizó una regresión lineal «paso a paso» para explicar el valor en el IMC a través del valor obtenido en la variable limitación de actividades. Por último se realizó una regresión lineal «paso a paso» para explicar el valor de la puntuación global en la CVRS a través del valor obtenido en las variables IMC, sedentarismo y cantidad de actividad física.

\begin{tabular}{|c|c|c|c|c|}
\hline Medidas* & $\begin{array}{c}\text { Niñ os con } \\
\text { sobrepeso (n= 29) }\end{array}$ & $\begin{array}{l}\text { Nños en normopeso } \\
(n=40)\end{array}$ & $p^{a}$ & $\begin{array}{l}\text { Tamaño del } \\
\text { efecto }\end{array}$ \\
\hline IMC & $26.36(2.02)$ & $20.89(1.91)$ & $<.001$ & 2.86 \\
\hline \multicolumn{5}{|l|}{ CVRS: PAQL(S) } \\
\hline Síntomas (puntos) & $5.17(1.10)$ & $5.54(.89)$ & .183 & -.37 \\
\hline Limitación de actividades (punt os) & $5.04(1.07)$ & $5.53(.99)$ & .049 & -.55 \\
\hline Función emocional (pu ntos) & $4.90(1.18)$ & $5.55(.89)$ & .023 & -.73 \\
\hline Puntuación global (puntos) & $4.82(1.32)$ & $5.54(.88)$ & .027 & -.81 \\
\hline \multicolumn{5}{|l|}{$\begin{array}{c}\text { ACTIVDAD FÍSICAY } \\
\text { SEDENTARISMO: IPAQ-A }\end{array}$} \\
\hline $\begin{array}{l}\text { Tiempo total de actividad } \\
\text { física/semana (minutos/semana) }\end{array}$ & $148.12(129.04)$ & $254.31(153.10)$ & .005 & .82 \\
\hline $\begin{array}{l}\text { Actividad física total/semana } \\
\text { (MET s/minuto/semana) }\end{array}$ & $563.07(574.38)$ & $979.17(666.26)$ & .005 & .72 \\
\hline $\begin{array}{l}\text { Tiempo total sentado/semana } \\
\text { (minutos/semana) }\end{array}$ & $118.97(71.93)$ & $98.50(70.34)$ & .043 & 1.54 \\
\hline
\end{tabular}

*Valores expresados como Med ia (DE); CVRS: Cal idad de vida relacionado con la salu d; PAQL (S): Cuestionario específico de Cal idad de vida relacionado con la sal ud en ni ños y jóvenes asmáticos; IPAQ-A:
Cuestionario Internacional de Actividad Física en niños y adolescentes.; $p^{a}$ : valor del estadístico p derivado del anál isis $\mathrm{t}$ de $\mathrm{S}$ tudent.

\section{Resultados}

En la tabla 1 se muestran los valores procedentes de las medidas tomadas en la muestra. Un total de 69 escolares con asma persistente controlado en nuestro estudio (donde 37 fueron chicos y 32 fueron chicas) cuya edad media fue de $14.47 \pm(1.64)$ fueron analizados en el presente estudio. En la tabla 2 se muestran los resultados procedentes del análisis de diferencias entre las medias de las medidas principales en 
Tabla 3. Correlación entre la CVRS y el nivel de actividad física y sedentarismo e IMCa (n=69) ACTIVDAD FÍSICA Y SEDENTARISMO:IPAQ-A

\begin{tabular}{|c|c|c|c|c|}
\hline $\begin{array}{l}\text { CVRS: } \\
\text { PAQL(S) }\end{array}$ & $\begin{array}{c}\text { Tiempo total de } \\
\text { actividad } \\
\text { física/semana } \\
\text { (minut os/semana) }\end{array}$ & $\begin{array}{c}\text { Actividad física } \\
\text { total/semana } \\
\text { (METs/minuto/s emana) }\end{array}$ & $\begin{array}{c}\text { Tiempo total } \\
\text { sentado/semana } \\
\text { (minutos/semana) }\end{array}$ & IMC \\
\hline $\begin{array}{l}\text { Sínt omas } \\
\text { (puntos) }\end{array}$ & -.062 & $\begin{array}{l}.047 \\
\end{array}$ & $-.500^{* *}$ & $\begin{array}{l}-.196 \\
\end{array}$ \\
\hline $\begin{array}{l}\text { Limi tación de } \\
\text { act ividades } \\
\text { (puntos) }\end{array}$ & -.043 & -.040 & $-.508^{* *}$ & $-.208 *$ \\
\hline $\begin{array}{l}\text { Función } \\
\text { emocional } \\
\text { (puntos) }\end{array}$ & .031 & .043 & $-346^{* *}$ & $-.211^{*}$ \\
\hline $\begin{array}{c}\text { Puntuaciớn } \\
\text { global (puntos) }\end{array}$ & 198. & -.004 & $-335^{* *}$ & $-.241^{*}$ \\
\hline IMC & $-315^{*}$ & $-.285^{*}$ & $.176 *$ & \\
\hline
\end{tabular}

Coeficientes de correlación de Pearson; CVRS: Calidad de vida relacionado con la salud; PAQL (S) Cuest ionano espectifico de Calidad de vida relacionado con la salu den niños y jóvenes asmáticos; IPAQ-A: Cren

ambos grupos tomadas para el estudio. Los niños con sobrepeso reportaron valores más elevados en cuanto al IMC que sus pares sin sobrepeso $(p<.001)$. En cuanto a la CVRS, los valores hallados en cuanto a las dimensiones referentes a la limitación de actividades $(p=.049)$, función emocional ( $p=.023$ ) así como en la puntuación global del cuestionario PAQL (S) $(p=.027)$ fueron significativamente mayores en niños con normopeso que en niños con sobrepeso. Además, los niños con sobrepeso auto reportaron un mayor nivel de sedentarismo $(p=.043)$ y un menor nivel de actividad física $(p=.005)$ que sus pares con normopeso En la tabla 3 puede observarse el nivel de correlación existente entre la CVRS y el nivel de actividad física y sedentarismo e IMC en los sujetos del estudio. El tiempo total de sedentarismo correlacionó de forma negativa con todas las dimensiones del cuestionario PAQL (S) al igual que el IMC. Éste último correlacionó de forma positiva con el tiempo de sedentarismo total. Sin embargo, correlacionó de forma negativa con el total de actividad física realizada a la semana. La varianza en los valores del IMC puede ser explicada en un 52\% a través de la varianza obtenida en el índice de limitación de actividades calculado en el cuestionario PAQL(S) (tabla 4), mientras que la varianza obtenida en la puntuación global obtenida en dicho cuestionario puede explicarse en un $31 \%$ por la varianza obtenida en los valores del IMC y del total de minutos de actividad física y sedentarismo (tabla 5).

Tabla 4. Regresión lineal explicati va del IMC en niños asmáticos (n=69).

\begin{tabular}{|c|c|c|c|c|}
\hline & \multicolumn{4}{|c|}{$\begin{array}{c}\text { IMC } \\
\text { Modelo }\left(\mathrm{R}=.72 ; \mathrm{R}^{2}=.52\right)\end{array}$} \\
\hline & ВЕТА & Eror típico & BETA estandarizada & $p^{a}$ \\
\hline $\begin{array}{l}\text { Limitación de } \\
\text { actividades }\end{array}$ & -.221 & .028 & -.722 & $<.001$ \\
\hline Constante & 10.754 & .678 & & $<.001$ \\
\hline
\end{tabular}
paciente; $p^{a}$ : valor estadístico de $p$ procedente del test ANOVA ajustado por edad y sexo.

Tabla 5. Regresión lineal explicativa del nivel de CVRS en niños asmáticos ( $\mathrm{n}=69$ ) CVRS: PAQL(S)

\begin{tabular}{ccccc}
\multicolumn{4}{c}{ Tabla 5. Regresión lineal explicativa del nivel de CVRS en niños asmáticos (n=69). } \\
\hline \multicolumn{4}{c}{$\begin{array}{c}\text { CVRS: PAQL(S) } \\
\text { Modelo }\left(\mathrm{R}=.55 ; \mathrm{R}^{2}=.31\right)\end{array}$} \\
\hline & BETA & Error típico & $\begin{array}{c}\text { BETA } \\
\text { estandarizada }\end{array}$ & $p^{a}$ \\
\hline $\begin{array}{c}\text { Tiempo total de actividad } \\
\text { física/semana (minut os/semana) } \\
\begin{array}{c}\text { Tiempo total sentado/semana } \\
\text { (minutos/semana) }\end{array}\end{array}$ & -.025 & .015 & -.189 & .027 \\
IMC & -.150 & .029 & -.543 & $<.001$ \\
Constante & -.352 & .638 & -.060 & .041 \\
& 157.513 & 14.590 & & $<.001$ \\
\hline
\end{tabular}

IMC: Índice de Masa Comoral; PAQL (S): Cuestionario de calidad de vida relacionado con la salud en niños asmáticos; $p^{a}$ : valor es tadístico de $p$ procedente del test ANOVA ajustado por edad y sexo.

\section{Discusión}

Los principales hallazgos de este estudio fueron dos. En primer se hallaron diferencias estadísticamente significativas entre niños en edad escolar con asma persistente controlados en lo referente a CVRS evaluada con el cuestionario PAQL(S), IMC y sedentarismo y cantidad de actividad física medidos en una semana mediante el cuestionario IPAQA. En segundo lugar se pudo determinar la relación que el IMC y sedentarismo (negativa) y de la cantidad de actividad física (positiva) tenían sobre la CVRS en la población estudiada. Hasta nuestro conoci- miento, este es el primer estudio en España que reporta datos referentes a la relación entre CVRS, actividad física, sedentarismo e IMC en niños en edad escolar que padecen asma.

Pese a que los participantes con normopeso obtuvieron una media de los valores en la puntuación global de la CVRS evaluada con el cuestionario PAQL(S) más alto que la media de los valores obtenida por sus pares con sobrepeso en la misma escala, ningún valor medio calculado estuvo por encima del punto de corte establecido para esta escala (Cano-Garcinuno, et al., 2007). Aunque no hemos encontrado estudios comparativos entre niños con normopeso y sobrepeso asmáticos, parece ser que los niños con asma presentan valores de sobrepeso más elevados que sus pares asintomáticos. La sintomatología en estos niños hace que la práctica deportiva se haga más difícil (Story, 2007). Lang et al, postula que los niños con asma tienen 3 veces más tendencia de realizar menos de 30 minutos diarios de $\mathrm{AF}$ que sus padres (Lang, Butz, Duggan, \& Serwint, 2004). Otros autores, en cambio, demuestran que no hay una reducción en la capacidad de realizar AF debido al asma (Lima, Santoro, Caetano, Cabral \& Fernandes, 2010; Santuz, Baraldi, Filippone \& Zacchello, 1997). La impresión clínica es que la obesidad es observada con mayor frecuencia en pacientes con asma que en la población sana. Los padres a menudo evitan que el niño participe en juegos activos o actividades que puedan prevenir la obesidad, por lo que muchos al final caen en sobrepeso u obesidad. Sin embargo, existen estudios que expresan que una reducción del IMC en niños asmáticos no mejoran sus síntomas (Fantuzzi, 2005), datos consistentes con los hallados en nuestro estudio en cuanto a la percepción de los síntomas en estos pacientes.

Por otro lado, desde los organismos de salud pública y educativos se entiende que maximizar la actividad física y minimizar el comportamiento sedentario es prioritario en edad infanto-juvenil, pero especialmente importante en asmáticos pues como hemos comentado con anterioridad, la obesidad en estos pacientes complica tanto el diagnóstico como el tratamiento (Vortmann \& Eisner, 2008). Pese a este consenso general, los niños y jóvenes con asma se encuentran con algunas barreras para la práctica de actividad física; de hecho, al menos el $90 \%$ de los individuos con asma presentan exacerbaciones tras la realización de ejercicio físico, provocando una limitación en la actividad (Juniper, 1997; Teuler, 2000). Acorde con estas afirmaciones, para poder explicar el valor del IMC en nuestros participantes, se estableció un modelo matemático regresivo con la variable que hipotéticamente más podría influir en dicho valor, la percepción de limitación de actividad. Por otro lado, existen creencias negativas acerca de lo que se puede o no se puede hacer en relación a actividad física, tanto por parte de pacientes, como de padres y médicos, lo que limita igualmente la participación de los pacientes en programas de actividad física. Consecuentemente, los jóvenes y niños con asma parecen estar en mayor riesgo de adopción de hábitos de vida sedentarios, pudiendo desembocar en sobrepeso u obesidad (Cheng et al., 2010). Esta afirmación es consistente con los resultados reportados en el presente estudio, donde hemos podido observar que existe una relación positiva entre los valores encontrados respecto del IMC y los valores reportados respecto del tiempo que los participantes en el estudio permanecieron sentados en una semana medido con el cuestionario IPAQ-A.

Este estudio presenta distintas limitaciones que han de ser tenidas en cuenta a la hora de interpretar los resultados. Por el tipo de diseño establecido, no es posible establecer una relación de causalidad entre las variables observadas por lo que futuros estudios longitudinales son necesarios para establecer dichas relaciones. De hecho, es necesario este tipo de estudios para establecer valores normativos de niños con asma para ver la relación de la CVRS y el nivel de actividad física y, a su vez, establecer qué nivel de actividad física es el que más favorece a la mejora de la CVRS en este tipo de población. La validez externa también necesita ser expuesta en este estudio. La muestra estuvo compuesta por niños asmáticos en edad escolar procedentes de la población sevillana, además la no se usaron técnicas de muestreo aleatorizadas, lo que puede limitar la generalización de los resultados. Se hacen necesarios estudios poblacionales para solventar esta cuestión. 


\section{Implicaciones prácticas}

El estudio provee de conocimiento de partida para el establecimiento de nuevas investigaciones en la línea de intervención en salud pública relacionada con el tópico estudiado en niños en edad escolar. Reducir al mínimo el sedentarismo y aumentar la actividad física es una conducta prioritaria para todos los niños y adolescentes, aunque más en aquellos sufrientes de asma por el aumento del riesgo de comorbilidad del asma y la obesidad. Por tanto, favorecer modelos lógicos de práctica deportiva en niños asmáticos en edad escolar es clave para el tratamiento y control de la enfermedad y el aumento de la CVRS de este tipo de población.

\section{Conclusión}

El valor del IMC puede afectar negativamente a la CVRS de los niños asmáticos en edad escolar, limitando la cantidad de actividad física que estos realizan y favoreciendo el sedentarismo en esta población.

\section{Referencias}

Ahmad, N., Biswas, S., Bae, S., Meador, K. E., Huang, R., \& Singh, K P. (2009). Association between obesity and asthma in US children and adolescents. J Asthma, 46(7), 642-646.

Bateman, E. D., Hurd, S. S., Barnes, P. J., Bousquet, J., Drazen, J. M., FitzGerald, M., et al. (2008). Global strategy for asthma management and prevention: GINA executive summary. Eur Respir J, 31(1), 143-178.

Beuther, D. A., Weiss, S. T., \& Sutherland, E. R. (2006). Obesity and asthma. Am J Respir Crit Care Med, 174(2), 112-119.

Brisbon, N., Plumb, J., Brawer, R., \& Paxman, D. (2005). The asthma and obesity epidemics: the role played by the built environmenta public health perspective. J Allergy Clin Immunol, 115(5), 10241028.

Cano-Garcinuno, A., Diaz-Vazquez, C., Carvajal-Uruena, I., PraenaCrespo, M., Gatti-Vinoly, A., \& Garcia-Guerra, I. (2007). Group education on asthma for children and caregivers: a randomized, controlled trial addressing effects on morbidity and quality of life. J Investig Allergol Clin Immunol, 17(4), 216-226.

Carvajal-Ureña, I., García-Marcos, L., Busquets-Monge, R., Morales Sánchez-Varela, M., \& García De Andoain, N. (2005). Variaciones geográficas de la prevalencia de síntomas de asma en los niños y adolescentes españoles. International Study of Asthma and Allergies in Childhood (ISAAC) Fase III. Arch Bronconeumol, 41, 659-666.

Carvajal-Uruena, I., Garcia-Marcos, L., Busquets-Monge, R., Morales Suarez-Varela, M., Garcia de Andoin, N., Batlles-Garrido, J., et al. (2005). [Geographic variation in the prevalence of asthma symptoms in Spanish children and adolescents. International Study of Asthma and Allergies in Childhood (ISAAC) Phase 3, Spain]. Arch Bronconeumol, 41(12), 659-666.

Cheng, B. L., Huang, Y., Shu, C., Lou, X. L., Fu, Z., \& Zhao, J. (2010). A cross-sectional survey of participation of asthmatic children in physical activity. World J Pediatr, 6(3), 238-243.

Fantuzzi, G. (2005). Adipose tissue, adipokines, and inflammation. $J$ Allergy Clin Immunol, 115(5), 911-919; quiz 920.

Ford, E. S. (2005). The epidemiology of obesity and asthma. J Allergy Clin Immunol, 115(5), 897-909; quiz 910.

Gilliland, F. D., Berhane, K., Islam, T., McConnell, R., Gauderman, W. J., Gilliland, S. S., et al. (2003). Obesity and the risk of newly diagnosed asthma in school-age children. Am J Epidemiol, 158(5), 406-415.

Hagstromer, M., Bergman, P., De Bourdeaudhuij, I., Ortega, F. B., Ruiz, J. R., Manios, Y., et al. (2008). Concurrent validity of a modified version of the International Physical Activity Questionnaire (IPAQ-A) in European adolescents: The HELENA Study. Int J Obes (Lond), 32 Suppl 5, S42-48.

Juniper, E. F. (1997). How important is quality of life in pediatric asthma? Pediatr Pulmonol Suppl, 15, 17-21.

Lang, D. M., Butz, A. M., Duggan, A. K., \& Serwint, J. R. (2004). Physical activity in urban school-aged children with asthma. Pediatrics, 113(4), e341-346.

Lima, P. B., Santoro, I. L., Caetano, L. B., Cabral, A. L., \& Fernandes, A. L. (2010). Performance of a word labeled visual analog scale in determining the degree of dyspnea during exercise-induced bronchoconstriction in children and adolescents with asthma. $J$ Bras Pneumol, 36(5), 532-538.

Lobstein, T., Baur, L., \& Uauy, R. (2004). Obesity in children and young people: a crisis in public health. Obes Rev, 5 Suppl 1, 4-104.

Santuz, P., Baraldi, E., Filippone, M., \& Zacchello, F. (1997). Exercise performance in children with asthma: is it different from that of healthy controls? Eur Respir J, 10(6), 1254-1260.

Schachter, L. M., Peat, J. K., \& Salome, C. M. (2003). Asthma and 7] atopy in overweight children. Thorax, 58(12), 1031-1035.

Story, R. E. (2007). Asthma and obesity in children. Curr Opin Pediatr, 19(6), 680-684.

Sucharda, P. (1989). [Clinical significance of the quantification of obesity. The Quetelet index and its use]. Cas Lek Cesk, 128(33), 10401043.

Tauler, E., Ferrer, M., Vall, O., \& Alonso, J. (2002). [Validation of the Spanish version of the Pediatric Asthma Quality of Life Questionnaire in children with asthma]. Med Clin (Barc), 118(11), 439.

Teuler, I. T. E. (2000). [Quality of life among pediatric asthma patients]. Allergol Immunopathol (Madr), 28(3), 168-175.

Vortmann, M., \& Eisner, M. (2008). BMI and health status among adults with asthma. Obesity (Silver Spring), 16, 146-152.
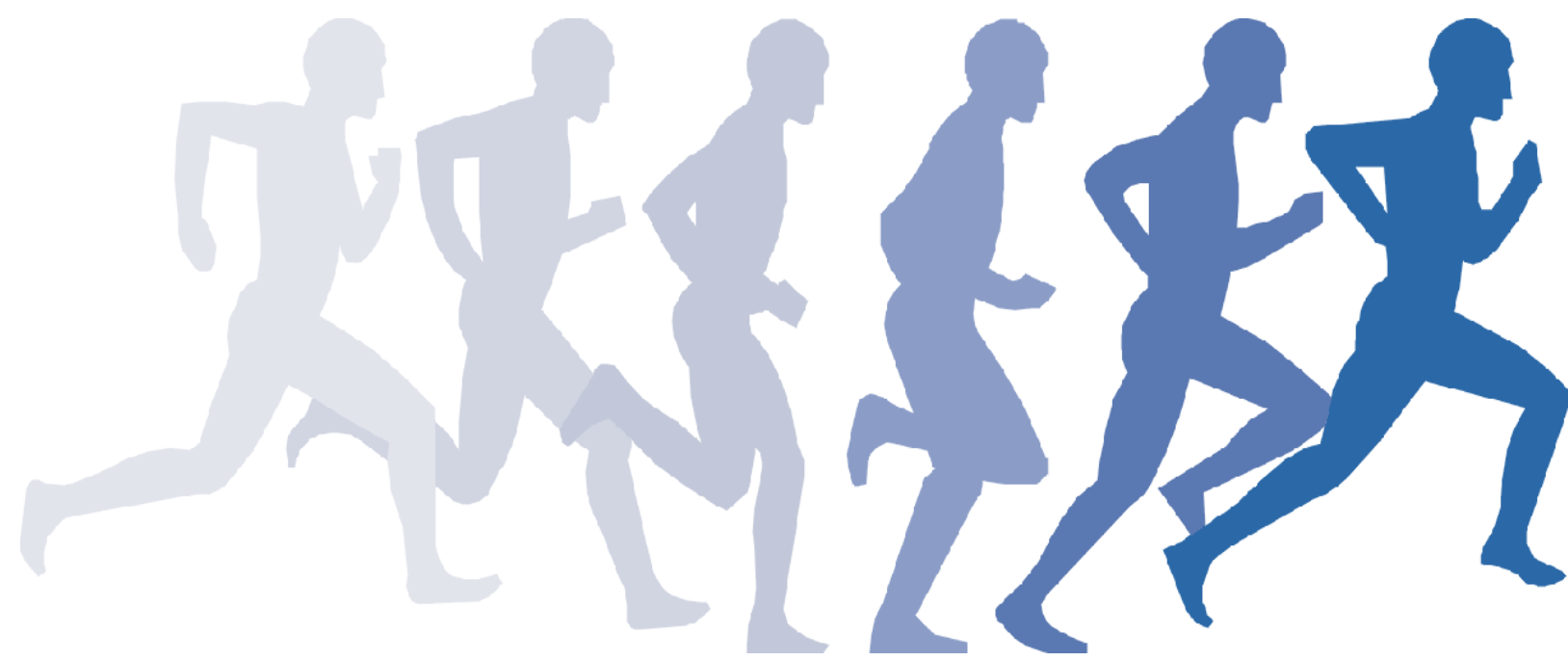